\title{
PAPR Reduction at Large Multi-User-MIMO-OFDM using Adaptive Data Detection Algorithm
}

\author{
N. Praba', K. M. Ravikumar ${ }^{2}$ \\ ${ }^{1}$ Department of ECE, GCE, Ramanagaram, India \\ ${ }^{2}$ SJCIT, chikkabalapur, India
}

\begin{tabular}{l} 
Article Info \\
\hline Article history: \\
Received Mar 8, 2018 \\
Revised Apr 27, 2018 \\
Accepted May 11, 2018 \\
\hline
\end{tabular}

Keywords:

Adaptive data detection (ADD) Multiple-input multiple-output (MIMO)

Orthogonal frequency-division multiplexing (OFDM)

Peak-to-average power ratio (PAPR)

\begin{abstract}
Wireless communication in present era contains large-scale MIMO network architecture that need to deliver an optimize-QoS to multi-user (MU). The optimize data rate transmission in massive MU-MIMO wireless systems is one of the most difficult task due to the extremely high implementation complexity. The practical wireless system channels generally exhibits the PAPR and frequency selective fading, it is also necessary to have a precoding solution in PAPR for the selected desirable channels. A solution for the designed problem of a noble error-correcting code for OFDM process with a low PAPR, in the case of impulse noise should be considered. In this paper, Adaptive-Data-detection (ADD) algorithm is proposed to obtain lowercomplexity data-detection that corresponds to high throughput design and impulse noise removal for large MUI-MIMO wireless systems by the OFDM modulation technique. That contains some steps such as; initialization, pre-processing and equalization steps in order to get no performance loss and to minimalize the recurrent amount at each iterations during operation. In order to use simplify model, here we assume suitably perfect synchronization, large cyclic prefix and perfect-CSI (channel-stateinformation) which has been developed through the pilot depended training. Simulation results analysis show the proposed method substantial improvement over the existing algorithm in terms of both 'Error-rate' minimization and PAPR reduction.
\end{abstract}

Copyright (C) 2018 Institute of Advanced Engineering and Science. All rights reserved.

\section{Corresponding Author:}

N. Praba,

Department of ECE,

GCE, Ramanagaram, India.

Email: npraba2010@ rediffmail.com

\section{INTRODUCTION}

Present wireless communication system contains large-scale MIMO network architecture that need to deliver a promising result in order to meet growing demands for multi-user quality-of-service $(Q o S)$ and higher throughput in wireless systems [1]. The large-scale MIMO network also should have ability to decrease the consumption of operational power $(O P)$ at the transmitter side, also support the usage of low complexity structures for concurring multi-user interface $(M U I)$. These kind of properties of large-scale MIMO makes a promising-technology for the upcoming generation of wireless systems. OFDM technique is well established and the attractive process to deal with the 'frequency selective' channels. Moreover, in order to simplify the receiver side equalization, OFDM process allows scheduling, bit allocation, and per-tone power in the spectrum shaping and frequency domain. The theoretical view of large-scale MIMO have acquired lot of significant attention from the researchers, but the knowledge of practical transmission process is very less. As per the paper [2], the practical operations of large-MIMO network will need the less radio frequency (RF) power and low cost components. In addition in [2], the proposed model of multi user precoding for flat-frequency channels depend on pre-antenna 'constant-envelope' transmission to allow the 
efficient operation through non-linear RF-components. The practical wireless system channels generally exhibits the PAPR and frequency selective fading, it also necessary to have a precoding solution of PAPR for selected desirable channels. The solution for such kind of complexity requires an individual mobile terminal to have less PAPR because of power constraints and stringent area; also, it can afford the heavy processing at the base station (BS).

However, the MIMO-OFDM is popular to undergo with a lesser PAPR that demands the usage of 'linear-RF' components (example, power amplifiers) in order to overcome the signal distortion and out-ofband radiation. Unfortunately, the linear-RF Component generally comes with less efficient power and more cost compared to the non-linear components, which causes the excessive cost for large-scale implementations of BS that may contains hundreds of antennas. In this paper [3], They proposed a residue number-system ('RNS') in order to reduce PAPR in MIMO-OFDM systems and the proposed model approach makes use of RNS properties to greatly decrease the 'computational complexity' as well as the PAPR. This methodology compared with the PTS-scheme (partial transmit sequence) also, where the RNS-based scheme of PAPR reduction performance can be analyze for computational complexity with minimal side information.

PAPR reduction approaches such as clip-signal has proposed in [4], [5], selected mapping (SLM) [6], [7] and partial transmit sequence (PTS) [8], [9] have been proposed in order to overcome the effects of PAPR in MIMO-OFDM signals. Therefore, using the techniques the 'PAPR' can be reduce, but these techniques also come up with their respective drawbacks. Clipping the peak amplitudes under a time domain reasons behind distortion of signal, which reduce the performance of BER in the receiver side. Partitions of PTS data into the sub-blocks, where individual sub-blocks are combined based on phase sequence and the phase sequences combination provides the lower PAPR at OFDM signal, which will be used at data transmission. The SLM combines phase sequences with their duplicates copy of OFDM data, and then performs several iterations of IFFT (inverse fast-Fourier-transform) to choose the block of data at lowest PAPR. Both of the method SLM and PTS require the side information transmission (in phase sequence) to recover the original transmitted data that may require some extra bandwidth. Therefore, it is necessary to reduce the amount of PAPR of OFDM dependent multi-user MIMO network system to provide corresponding low-power and low-cost BS operations. Moreover, the MUI cancelation can be given as underdetermined linear regularization problem that requires optimize solution.

In this paper, we proposed a lower-complexity data-detection algorithm that corresponding to high throughput design for large MUI-MIMO wireless systems by the OFDM modulation technique. DDA (Data detection algorithm) can improve the computational efficiency in a hardware prototype that avoids the excess computation. A DDA is a well-known iterative framework to solve a huge number of convex difficulty (exactly or approximately) through coordinate-wise updates, but here we proposing Adaptive-Datadetection (ADD) algorithm that contains the initialization, pre-processing and equalization steps in order to get low performance loss, minimization of impulse noise and minimalize the recurrent amount at each iterations during operation. In order to use simplify model, we assume suitably perfect synchronization, large cyclic prefix and perfect-CSI is been developed through the pilot depended training. This algorithm performs through considered regularization parameters, which enables the neighboring maximum likelihood values that correspond to performance of output data detection in large MU-MIMO systems, moreover the large 'BS-to-user-antenna' ratio. Simulation results analysis show the proposed method substantial improvement over the existing algorithm in terms of both 'Error rate' minimization and PAPR reduction.

The paper is organised as follows; Section 2 represents literature survey, Section 3 shows the proposed system model, Section 4 represent the experimental result and analysis, and Section 5 conclude our work.

\section{LITERATURE SURVEY}

Inspite of many advantages of MIMO-OFDM signals, the high PAPR value is main drawback that coming in signals. In order to decrease the high PAPR, there are many PAPR reductions approach have been proposed by the researchers and in this section we are going elaborate the different PAPR reduction technique and the problem associated with it.

The selected mapping (SLM) approach is an efficient scheme because of its high PAPR reduction capability without the distortion of signal, while the conventional SLM ('CSLM') approach come up with major complexity in computation. There are several methodologies has been proposed to overcome the difficulty, ABC (Artificial Bee Colony) technique is a newly proposed swarm based optimization approach which provide the high performance in regarding of PAPR minimization. The performance of ABC approach generally based upon the bees search strategies and there are also some modified search strategies are there. In paper [10], a parallel $\mathrm{ABC}(\mathrm{P}-\mathrm{ABC})$ has proposed to modify the search strategy, and improved (I-ABC) approach is been introduced to decrease the complexity in computation at SLM approach. A PABC follows 
strategies to obtain best exploitation and exploration. In I-ABC, an individual bee search for the optimal neighborhood to obtain best solution for mutation process. Afterwards, the obtained solutions is shared with different swarm and novel 'search directions' is obtained to find optimum sources.

In (single-input single-output) SISO-OFDM wireless systems, the best projecting techniques are clipping [11], active constellation extension ('ACE') [12], tone reservation (' $T R$ ') [13], partial transmission sequence ('PTS') [14], selected mapping ('SLM') [15], and others. In [16]; they provided a detailed overview, in which the PAPR-reduction approach can be stretched easily from SISO to MIMO systems [17], [16], the extension of multiuser (MU) MIMO system is not that much easy, because the joint process of receiver-side signal transmission is almost very difficult in practical at presence of distributed users. MIMO-OFDM system has been extensively accepted to serve for the 'wireless communication' systems. Though it is still undergoes from the maximal PAPR that is the main disadvantage of MIMOOFDM-based systems.

In paper [18], they consider the approach to reduce the PAPR in coded OFDM systems. A novel PAPR minimization approach using the 'label-inserted' encoder of soft amplitude limiter (' $S A L$ ') and using this approach they got 5.5dB PAPR minimization (in OFDM system), which contain 3dB clipping, 128 subcarriers, and 4-bit selection. Excluding the significance of PAPR minimization this approach also have advantage of low complexity, small overhead, small performance loss, and no extra information transmission. Amongst several random process, an irregular repeat accumulate ('IRA') code is finest option for simpler encoder process. The prototype can be applied directly to MIMO-OFDM system and the capacity of clip MIMO-OFDM can be analyze through Gaussian approximation. Here, they consider soft MAP detector with iterative receiver in MIMO-OFDM system. In different number of antennas systems, the hybrid approach is become independent part of encoder, therefore there is no extra constraint has applied at IRAcode optimization. The designing of IRA codes is complete according to the ergodic MIMO system by consider several PAPR decrement setting, moreover, the different clipping ratios is based on extrinsic information transfer ('EIT') plans. The high value of PAPR leads to the in-band distortion along with frequency spread spectrum, due to the presence of non-linearity in the 'high-power' amplifiers and the larger PAPR value essential to decrease although its probability should not much high. While eliminating the high peaks the signal performance is also affected, therefore, a special maintenance needs to be consider to decrease the PAPR in MIMO-OFDM. In this paper [19], they proposed a novel algorithm that is based upon the grouping of PTS and SLM methods for the PAPR reduction. In previous existing techniques of PAPRreduction only uses either PTS or SLM as a separate technique on MIMO-OFDM. Here, they consider QPSK modulated technique in MIMO-OFDM systems through considering four number of antennas.

In [20] and [21] proposed a Pulse shaping method that is effective in decreasing the PAPR of MIMO-OFDM signal, also provide the lower computational complexity. Selecting the proper pulse, the PAPR can be reduced, moreover the Nyquist-pulses as 'square-root raised cosine' (SRRC) and raised cosine (RC) are usually used pulses. Conversely, the ideal Nyquist-pulses are normally non-causal, that is why it is not practically reachable and the ideal Nyquist-pulses can be shorten at time domain to preserve the causality, but this model introduces the undesired side lobes of non-zero in frequency domain. To design a fundamental pulse using an efficient computational optimization method [22], which offered a much effective method for executing pulse shaping method in MIMO-OFDM systems in order to decrease the high PAPR values. However, this pulse-shaping approach may present inter-carrier interference (' $I C I$ ') and eliminate the orthogonality function in the MUI-MIMO-OFDM system. Therefore, it causes the degradation in the performance of demodulation in OFDM system [23].

There are always some residual that leading to signal-to-interference and noise ratio. To overcome this problem, in [24] they proposed a zero-forcing equalization technique. The advancement in communication field outcome require the higher data rates with lower bit-error-rate (BER) and increase in the power efficiency. MIMO-OFDM is comprehensively use in the present and subsequent generation broadband or wireless communications [25] to generate a higher data-rate transmission, also the spectral efficiency at some multipath channels fading. The MIMO systems having the major drawback, which suffers from the high PAPR that requires implementation of highly efficient power amplifier, in order to continue a broader linear region for avoiding the signal clipping, therefore it increases the power consumption and hardware complexity. The several reduction technique of PAPR has been proposed that provide the maximal reduction of PAPR, which ranging from $0.5 \mathrm{~dB}$ to $6 \mathrm{~dB}$. In paper [26], discussed the PAPR performance analysis, also the algorithms for beam-forming (BF) techniques that include MRT (Maximum Ratio Transmission), EGT (Equal Gain Transmission) and combining of receiver techniques such as MRC (Maximum Ratio Combining), EGC (Equal Gain Combining) for MIMO-OFDM system. Moreover, the MIMO-OFDM system measurement is complete in terms of PAPR reduction, SNR and BER. 


\section{PROPOSED SYSTEM MODEL}

In this section, we consider a large MUI-MIMO-OFDM system to address the MUI cancellation and PAPR reduction. Precisely, the MUI cancelation can be given as underdetermined linear regularization problem that requires optimize solution. In MIMO-OFDM model, $A$ is an antenna user terminals that transfer the data simultaneously BS-antenna $C \gg A$ over the $B$ subcarriers and the user encodes its personal bit stream $(i=1, \ldots, A)$ through forward error correction approach. Afterwards, the coded bits signal onto gathering point at a $D$ finite set i.e., 16-QAM, with average transmit power in unity $F\left[|e|^{2}\right]=1$ at $G=\log _{2}|H|$ (i.e., bits/constellation point) and $e \in H$.

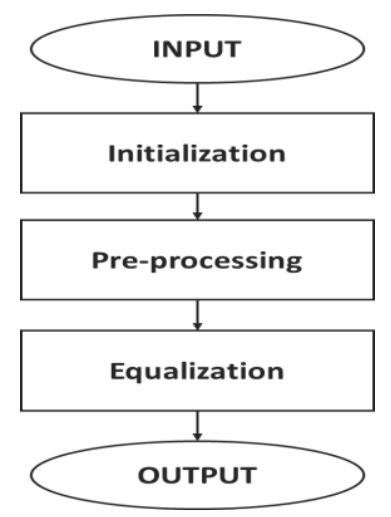

Figure 1. Flowchart of Proposed Model

Then the resulting frequency domain $(B)$ symbols $\left[e_{1}^{i}, \ldots, e_{b}^{i}\right]$ are transformed in a time domain symbol using IDFT [27] (inverse discrete 'Fourier Transform'). After pre-pending cyclic start, all the users transfer their time domain signals at the selective 'wireless frequency channel' at same period. After, discarding cyclic prefix, the time domain signals has received in each of the BS-antennas and then transformed into frequency domain using discrete 'Fourier Transform'. Figure 1 shows the flowchart of proposed model.

In order to use simplify model, we assume suitably perfect synchronization, large cyclic prefix and perfect-CSI (channel-state information) is been developed through the pilot depended training. Considering this assumption, the frequency domain relation of input and output with bth subcarrier can be modeled as;

$$
k_{b}=L e_{b}+m_{b}
$$

Where, the $k_{b} \in Y^{C}$ can be associated with frequency domain received vector, $e_{b} \in H^{A}$ contains the transmitted symbol through all users $(A)$ and channel matrix is represented as;

$$
k_{b} \in Y^{C} \in Y^{C \times A}
$$

The symbols is transmitted thru user $i$ at $b$-subcarrier and thermal noise modeled as $m_{b} \in Y^{A}$ at symmetric complex Gaussian vector with noise variance $M$ and impulse noise.

In practical, the underdetermined linear regularization problem can be solve by regularize the leastsquare difficulty;

$$
e_{b}^{e r r}=\underset{r \in Y^{A}}{\operatorname{argmin}}\left\|k_{b}-L_{b} r\right\|_{2}^{2}+M\|r\|_{2}^{2}
$$

Since the Equation (3) is quadratic form in r, so the regularization parameter problem has in closed form solution. While the problem occur in above Equation (3) can be calculated implicitly through data-detection algorithm (DDA). DDA can improve the computational efficiency in a hardware prototype that avoids the excess computation. A DDA is a well-known iterative framework to solve a huge number of convex difficulty (exactly or approximately) through coordinate-wise updates and we can define the function as;

$$
f\left(r_{1}, \ldots, r_{A}\right)=f(r)=\left\|k_{b}-L_{b} r\right\|_{2}^{2}+p(r)
$$


Where, the $p(r)$ is 'convex regularizer' and it is necessary to realize the Equation (3), while minimizing Equation (4) through selecting $p^{e r r}(r)=M\|r\|_{2}^{2}$. Here, minimizing is similar to solve the square error regularization problem through,

$$
p^{o}(r)=S(r \in Q)
$$

Where, $S(r \in Q)$ define the functional characteristics that is zero if the $r \in Q$ and otherwise it is infinity.

Minimizing Equation (5) based equalization function evolves $f\left(r_{1}, \ldots, r_{A}\right)$ sequentially to coordinate each variable $r_{A}$ and $a=1, \ldots, A$.

Here, we assume DDA based square error equalization to find ath optimum rate $r_{a}$ for error equalization problem in (3).

$$
\hat{r}_{a}=\underset{r \in Y}{\operatorname{argmin}}\left\|k_{b}-L_{b} r\right\|_{2}^{2}+M\|r\|_{2}^{2}
$$

Which holds the other values of $r_{j}, \forall j \neq a$ fixed. This involved a quadratic problem and it can solve through setting the function of gradient in (6) w.r.t the ath component to zero.

$$
0=\nabla_{a} f(r)=l_{a}^{L}(L r-k)+M r_{a}
$$

Through decomposing Equation (8), the Equation (7) for $r_{a}$ in order to get closed from expression:

$$
\begin{aligned}
& L r=\sum_{j \neq a} l_{j} r_{j}+\mathrm{l}_{a} r_{a} \\
& \hat{r}_{a}=\left[k-\sum_{j \neq a} \mathrm{l}_{j} r_{j}\right]^{l_{a}^{L}} /\left(\left\|l_{a}\right\|_{2}^{2}+M\right)
\end{aligned}
$$

The expression is accurately update DDA rule for ath entry of $r$, and for each iteration, Equation (9) can calculate sequentially for user $a=1, \ldots, A$, where frequently reuse all new novel outcome $\hat{r}_{a}$ for $a t h$ user. Therefore, we repeat the process for $T$ total number of iteration to estimate;

$$
\tilde{e}^{e r r}=r^{(T)}
$$

Where, $r^{(T)}$ is the final result of DDA based described iterative process.

DDA enables a non-linear data-detection approach, which operate openly in 'frequency domain' on each subcarrier basis. This update derive the box-constrained equalization problem. Moreover, the characteristics function can be provide through (5) is not differentiable, but it is similar method that can be use sub-gradients to enables closed form of expression,

$$
\hat{r}_{a}=o p_{Q}\left(\frac{k \times l_{a}^{L}-k \sum_{j \neq a} 1_{j} r_{j}}{\left\|h_{u}\right\|_{2}^{2}}\right)
$$

Here, $o p_{Q}(\cdot)$ shows the orthogonal projection at the $Q$ convex polytope and given by;

$$
o p_{Q}(b)=\left\{\begin{array}{cc}
b & \text { if } b \in Q \\
\arg \min _{g \in Q}|b-g| & \text { if } b \notin Q
\end{array}\right.
$$

The argument $b \in q$ is under the set of $Q$, and then $b$ is projection outputs; the $b$ is external set of $Q$ in the Euclidean distance. There are several relevant constellation $H$ sets, and (12) projection can be obtain effectively for QAM constellation. Where, imaginary and real part can be clip independently of $w$ onto the $[-U,+U]$ interval and $U$ is a radius of close-fitting box, which covers the 'QAM' constellation.

In this paper, we proposing Adaptive data-detection (ADD) algorithm that contains the initialization, preprocessing and equalization steps in order to get no performance loss and to minimalize the recurrent amount at each iterations during operation $(t=1, \ldots, T)$. Instead, to compute blindly updates in (9) and (11) for regularization parameter, the preprocessing and restructuring step are performed. In preprocessing is performed to decrease the operational complexity, ADD pre-calculate the several major quantities, which can be reprocessed at individual iteration of $t=1, \ldots, T$. This type of preprocessing is not only provide the significant saving during complexity at iterative process (such as DDA), but also simplifies the hardware implementations. 
In particular way, we pre-calculate regularized (inverse squared column) norms of $L$, that is;

$$
v_{a}^{-1}=\left(\left\|l_{a}\right\|_{2}^{2}+U\right)^{-1}
$$

Where, $a=1, \ldots, A$, with $U \geq 0$ and the regularized parameter gain is;

$$
w_{a}=v_{a}^{-1}\left\|l_{a}\right\|_{2}^{2}
$$

In the error mode of regularization parameter $U=M$ and in box-constrained regularization mode $U=0$, that yields $w_{a}=1, a=1, \ldots, A$.

To avoid the recurrent process at equalization operation, ADD algorithm provide the incremental updates that can reuse intermediate quantities at each phase of iteration $t=1, \ldots, T$. Therefore, we performing sequential upgradation, also can be called as approximate residual vectors and defined as;

$$
z=k-\sum_{j=1}^{A} l_{j} r_{j}^{(t)}
$$

However, we are not going to re-calculate the approximate residual vectors for individual iteration. In conflicting, we initially compute the symbol estimation $r_{a}^{(t)}$ by the approximate residual vectors.

$$
r_{a}^{(t)}=o p_{q}\left(v_{a}^{-1} l_{a}^{L}+r_{a}^{(t-1)} \times w_{a}\right)
$$

The delta value of equation (16) can be compute as;

$$
\Delta r_{a}^{(t)}=r_{a}^{(t)}-r_{a}^{(t-1)}
$$

Equation (17) enables the $(z)$ residual update in (18) without computing the residual explicitly.

$$
z \leftarrow z-\Delta r_{a}^{(t)} l_{a}
$$

The received output vector can be computed as;

$$
\tilde{e}=\left[r_{1}^{(t)}, \ldots, r_{A}^{(t)}\right]^{T}
$$

As we described above, ADD algorithm provide the significantly less computational complexity. In DDA approach, it requires a complex inner product value and $(A-1)$ scalar-by-vector complex multiplications at per iteration of $t$, whereas the proposed ADD approach requires only one scalar-by-vector complex multiplication and one inner product value.

\section{EXPERIMENTAL RESULTS AND ANALYSIS}

The simulation is part is done using Matlab 2016b, system configuration; 8GB RAM, 2GB graphics card, 1TB ROM and intel i5 processor. In order to evaluate the performance of error-rate for our proposed ADD model with respect to Zero Forcing [24] algorithm, we performing 'Monte-Carlo' simulations in MIMO-OFDM system, where LTE Advanced (LTE-A) technique is used for data transmission with the subcarriers [28]. In this study, we use 16-QAM with gray mapping and to account the frequency and spatial correlation we create channel estimation matrices using Winner-Phase-2 prototype [29] with considering $7.8 \mathrm{~cm}$ antenna distance (spacing). We report the symbol error rate (SER), Bit error rate (BER) and peak-toaverage power ratio ('PAPR') is consider as peak amplitude squared value divided by 'root mean square' in order to get average power. The BER and SER performance of 16-QAM arrangement with OFDM in presence of Gaussian noise and impulsive noise will deliver the system performance using our proposed ADD model with respect to Zero Forcing algorithm.

Here, we considering two scenarios where we fix the number of receive antennas and varying the transmit antennas using 16QAM modulation technique. Monte-Carlo trials is consider as 10000 and the signal-to-noise-ratio (SNR) is varying from -10 to $10 \mathrm{~dB}$ (with the interval of $2 \mathrm{~dB}$ ). In scenario-a, we consider 32 number of receive antennas with 16 transmit antennas and, 32 number of receive antennas with 32 transmit antennas in order to evaluate the error rate and PAPR. Similarly in scenario-b, we consider 64 number of receive antennas with 32 transmit antennas and, 64 number of receive antennas with 64 transmit 
antennas in order to evaluate PAPR and the error rate. Through varying the antennas number at SNR, we can analyze the behavior of our proposed model.

Figure 2 shows for the SNR vs SER at 32 number of receiving antenna (RAs) and 16 transmitting antennas (TAs), where our proposed model perform considerably well with increasing number of SNR, in 10dB SNR the ADD got 6.17\% less SER than the ZF[24]. Similarly, in Figure 3 shows for the SNR vs SER at 32 RAs and 32 TAs, where the SER is little more compare to Figure 2, whereas our proposed model got $41 \%$ less SER at 10dB SNR. Figure 4 shows for the SNR vs BER at 32 RAs and 16 TAs, at 10dB SNR our proposed model ADD performs 6.6\% less compared to the ZF approach. Similarly, Figure 5 shows for the SNR vs BER at 32 RAs and 32 TAs, where in $-10 \mathrm{~dB}$ ZF got $11 \%$ more BER compared to ADD. Figure 6 and 7 shows for the SNR vs PAPR at 32 RAs with 16 and 32 TAs; considering 10dB SNR in Figure 6 ADD got $1.25 \%$ less PAPR then ZF and in Figure 7 ADD got $24 \%$ less PAPR then ZF approach. Below Figure 8 to 13 shows for the scenario b; where Figure 8 and 9 shows for the SNR vs SER at 64 RAs with 32 and 64 TAs, Figure 10 and 11 shows for the SNR vs BER at 64 RAs with 32 and 64 TA. Moreover, Figure 12 and 13 shows for the SNR vs PAPR at 64 RAs with 32 and 64 TAs, where in Figure 12, proposed ADD shows the $1.5 \%$ less PAPR at 10dB SNR and in Figure 13, proposed ADD shows the 35\% less PAPR at 10dB SNR. Table 1 show for PAPR value at different SNR per received antennas [dB], where considered total number of receiving antennas is 128 and considered transmit antenna is 64. In -2dB SNR, ADD proposed model got $23 \%$ less PAPR value compared to ZF approach and in 4dB SNR, ADD model got 7.7\% less PAPR value compared to ZF approach. Therefore, from the analysis of result we can say that increasing in SNR per received antennas the average value of PAPR is decreasing.

- $\quad$ Scenario- $a$

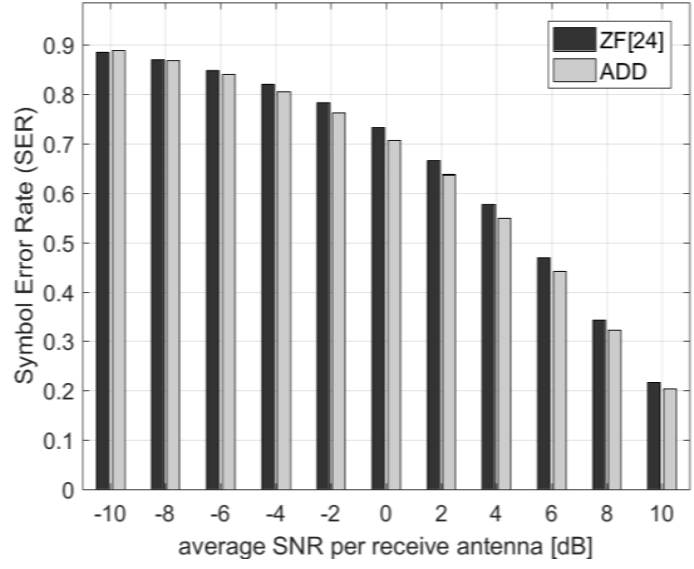

Figure 2. SNR vs SER (RAs=32 and TAs=16)

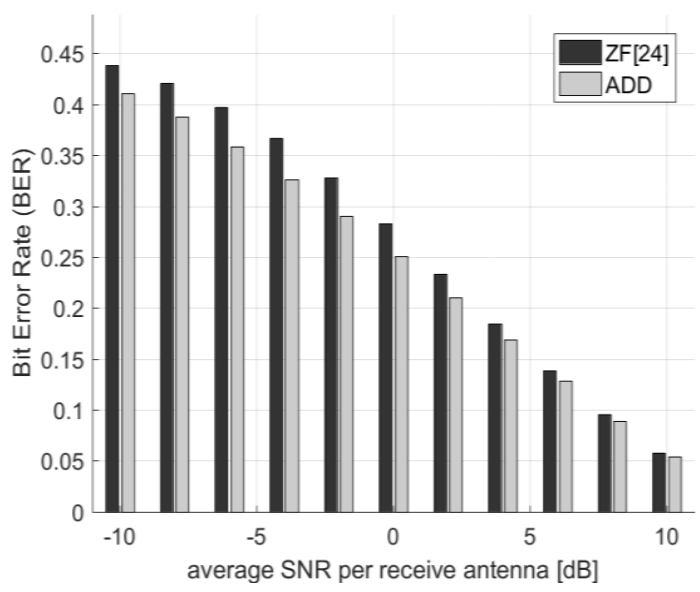

Figure 4. SNR vs BER (RAs=32 and TAs=16)

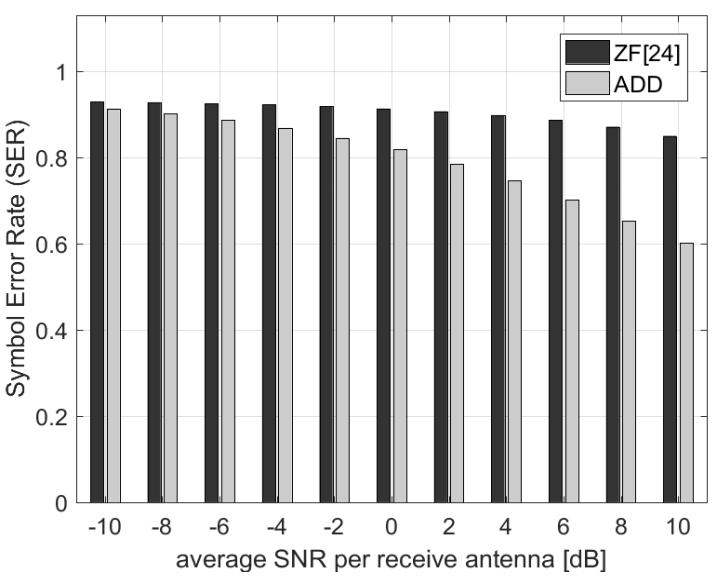

Figure 3. SNR vs SER (RAs=32 and TAs=32)

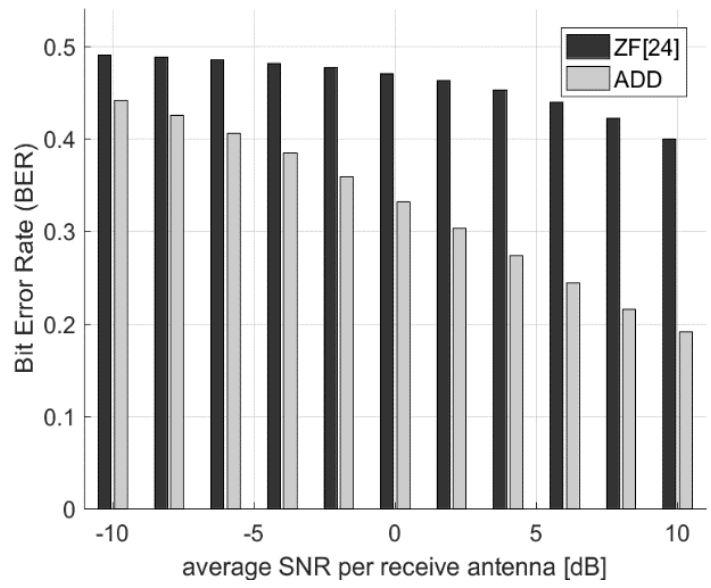

Figure 5. SNR vs BER (RAs=32 and TAs=32) 


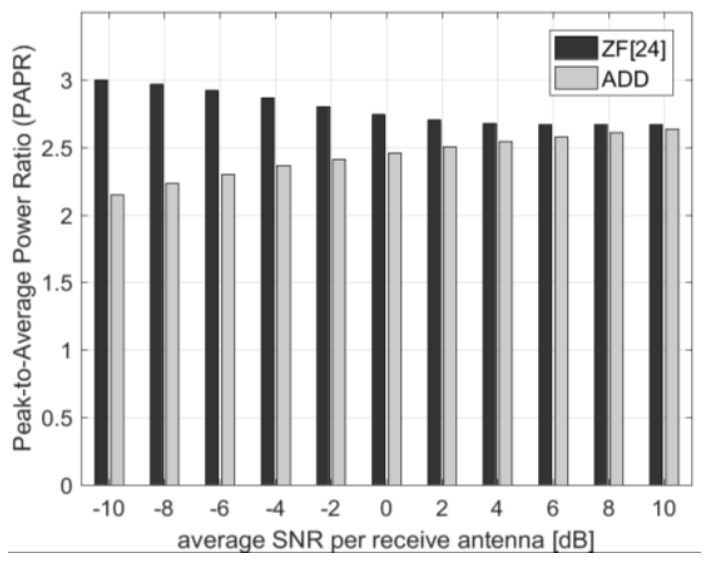

Figure 6. SNR vs PAPR $(\mathrm{RAs}=32$ and TAs=16)

\section{- Scenario-b}

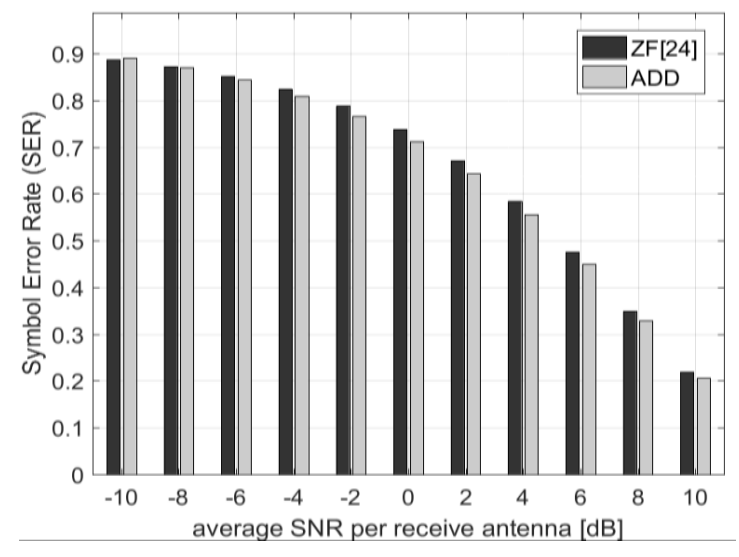

Figure 8. SNR vs SER (RAs=64 and TAs=32)

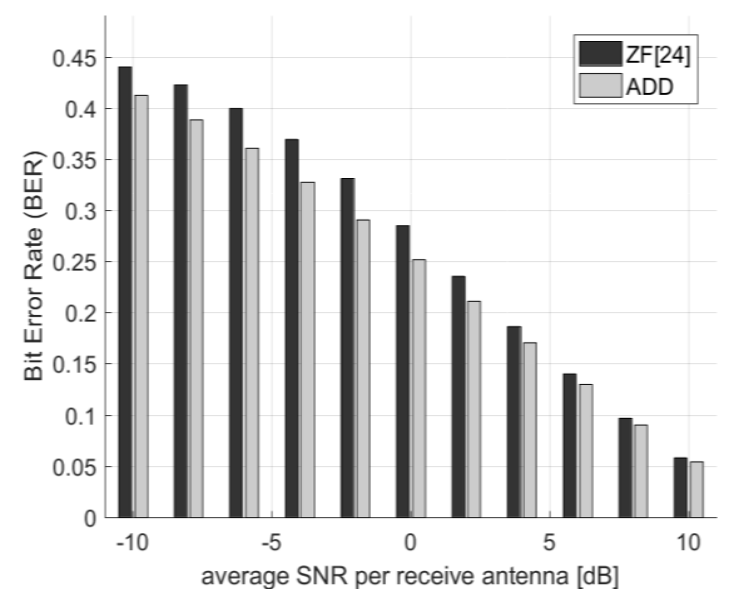

Figure 10. SNR vs BER (RAs=64 and TAs=32)

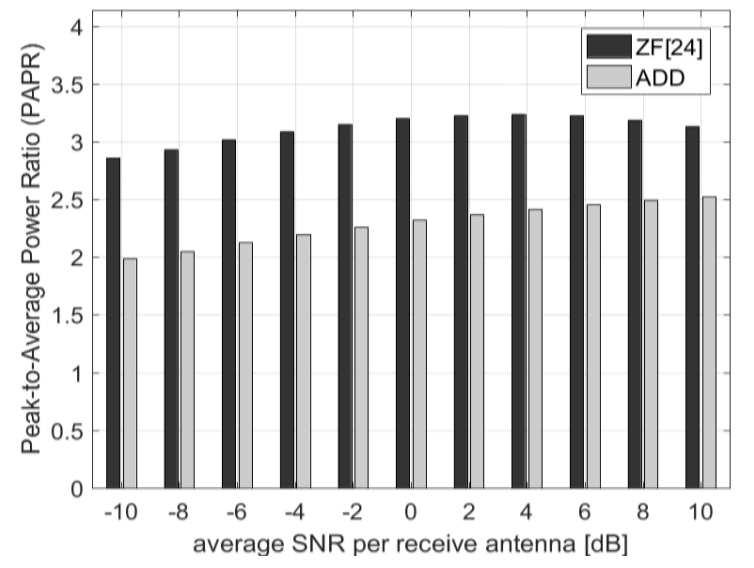

Figure 7. SNR vs PAPR (RAs=32 and TAs=32)

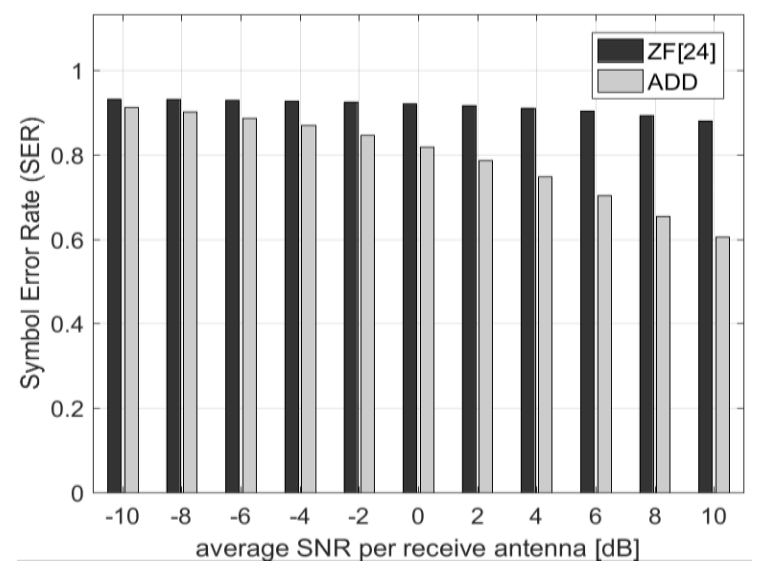

Figure 9. SNR vs SER (RAs=64 and TAs=64)

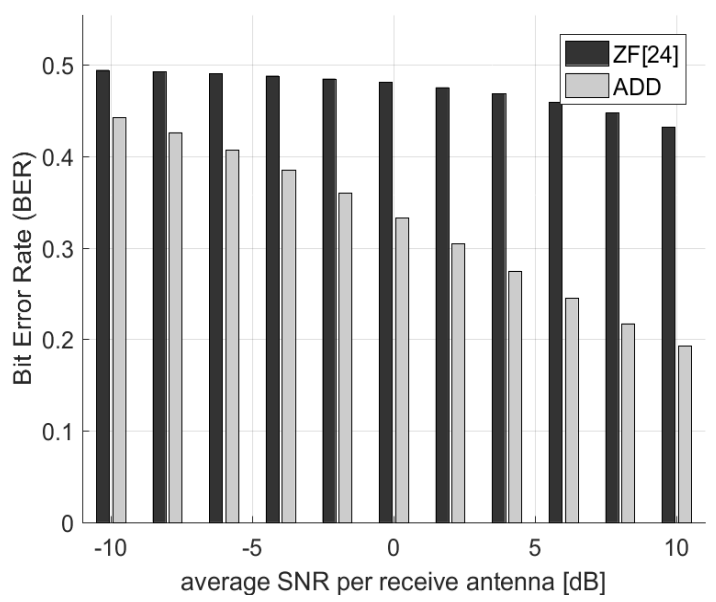

Figure 11. SNR vs BER (RAs=64 and TAs=64) 


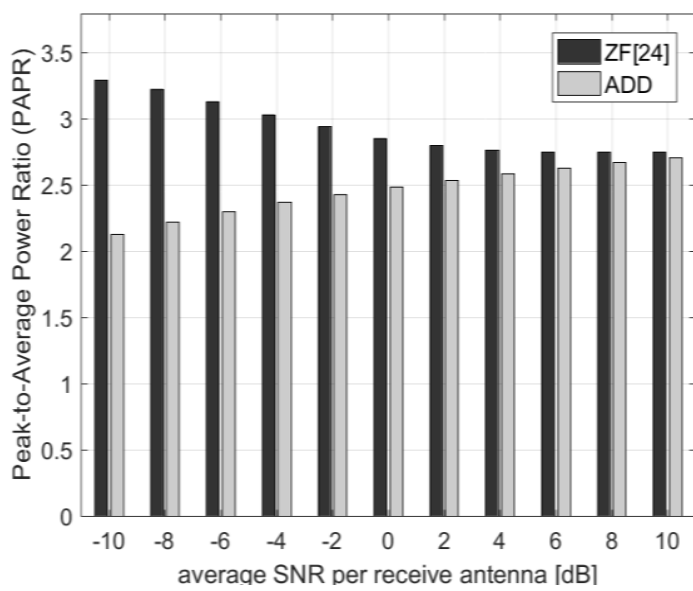

Figure 12. SNR vs PAPR (RAs=64 and TAs=32)

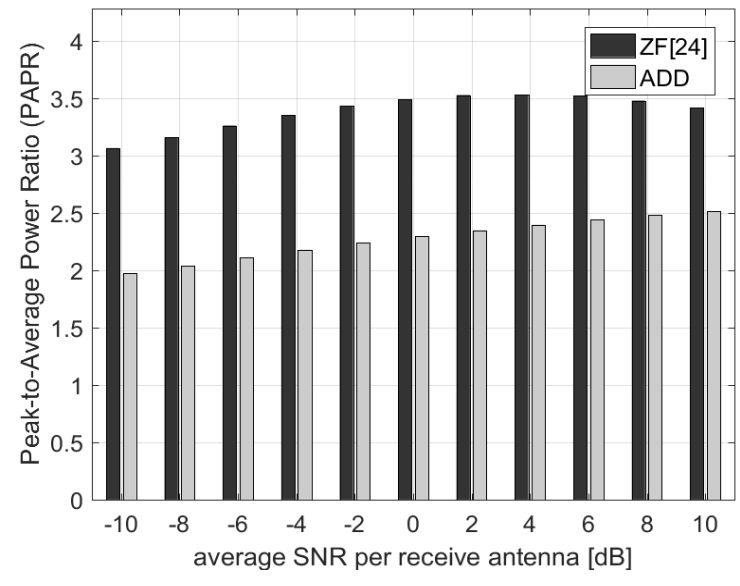

Figure 13. SNR vs PAPR (RAs=64 and TAs=64)

Table 1. PAPR value at different SNR (RAs=128 and TAs=64)

\begin{tabular}{|c|c|c|c|c|c|c|c|c|c|c|c|}
\hline SNR[dB] & -10 & -8 & -6 & -4 & -2 & $\mathbf{0}$ & 2 & 4 & 6 & 8 & 10 \\
\hline $\mathrm{ZF}[24]$ & 3.47 & 3.36 & 3.24 & 3.11 & 2.98 & 2.88 & 2.81 & 2.77 & 2.76 & 2.76 & 2.76 \\
\hline ADD & 2.11 & 2.19 & 2.28 & 2.35 & 2.41 & 2.47 & 2.52 & 2.57 & 2.63 & 2.67 & 2.71 \\
\hline
\end{tabular}

\section{CONCLUSION}

We have proposed Adaptive data detection (ADD) algorithm based detection technique for the large MUI-MIMO system by the use of orthogonal-frequency-division-multiplexing ('OFDM') with the presence of Impulse and Gaussian noise. This proposed ADD algorithm having both linear and non-linear regularization constraint to provide simple detection operation. Here, we considered two scenarios; scenario$a$ and scenario- $b$ to validate the performance of our proposed model. Where we fix the number of receive antennas and varying the transmit antennas using 16QAM modulation technique and 10000 Monte-Carlo trials has been taken at different signal-to-noise-ratio (SNR). Our results shows that ADD is suitable for practical OFDM based large MUI-MIMO system to support several users communicating with hundreds of base stations by achieving better data transaction rate and PAPR reduction with presence of Impulse and Gaussian noise. In considering 128 RAs 128 and 64 TAs, we got 1.8\% reduction in PAPR at 10dB SNR. In future work, the ADD model can be implement in VLSI for faster convergence and can enable high transmission at same PAPR.

\section{REFERENCES}

[1] F. Rusek, D. Persson, B. K. Lau, E. G. Larsson, O. Edfors, F. Tufvesson, and T. L. Marzetta, "Scaling up MIMO: opportunities and challenges with very large arrays," arXiv:1201.3210v1, Jan. 2012.

[2] S. K. Mohammed and E. G. Larsson, "Per-antenna constant envelope precoding for large multi-user MIMO systems," arXiv:1111.3752v1, Jan. 2012.

[3] Hala M. Abd Elkader, Gamal M. Abdel-Hamid, Adly Tag El-Dien, Asmaa A. Nassif, "Combined Beamforming with Orthogonal Space Time Block Code for MIMO-OFDM with Simple Feedback", Indonesian Journal of Electrical Engineering and Computer Science Vol. 4, No. 3, Desember 2016, pp. 580 585 DOI: 10.11591/ijeecs.v4.i3.pp580-585.

[4] X.LiandL.Cimini,"Effects of clipping and filtering on the performance of OFDM," Communications Letters, IEEE, vol. 2, no. 5, pp. 131-133, May 1998.

[5] X. Zhu, W. Pan, H. Li, and Y. Tang, "Simplified approach to optimized iterative clipping and filtering for PAPR reduction of OFDM signals," IEEE Transactions on Communications, vol. 61, no. 5, pp. 1891-1901, May 2013.

[6] S. Umeda, S. Suyama, H. Suzuki, and K. Fukawa, "PAPR reduction method for block diagonalization in multiuser MIMO-OFDM systems," in Vehicular Technology Conference (VTC 2010-Spring), 2010 IEEE 71st, May 2010, pp. 1-5.

[7] Kalvein Rantelobo, Hendro Lami, Wirawan, "Video Transmission using Combined Scalability Video Coding over MIMO-OFDM Systems", Indonesian Journal of Electrical Engineering and Computer Science Vol. 4, No. 2, November 2016, pp. 390 396 DOI: 10.11591/ijeecs.v4.i2.pp390-396.

[8] S. H. Han and J. H. Lee, "An overview of peak-to-average power ratio reduction techniques for multicarrier transmission," Wireless Communications, IEEE, vol. 12, no. 2, pp. 56-65, April 2005.

[9] J. C. Chen, "Partial transmit sequences for PAPR reduction of OFDM signals with stochastic optimization techniques," IEEE Transactions on Consumer Electronics, vol. 56, no. 3, pp. 1229-1234, Aug 2010. 
[10] N. Taşpınar and M. Yıldırım, "A Novel Parallel Artificial Bee Colony Algorithm and Its PAPR Reduction Performance Using SLM Scheme in OFDM and MIMO-OFDM Systems," in IEEE Communications Letters, vol. 19, no. 10 , pp. $1830-1833$, Oct. 2015.

[11] R. ONeill and L. B. Lopes, "Envelope variations and spectral splatter in clipped multicarrier signals," in Proc. IEEE PIMRC'95, Toronto, Canada, Sep. 1995, pp. 71-75.

[12] B. S. Krongold and D. L. Jones, "PAR reduction in OFDM via active constellation extension," IEEE Trans. Broadcasting, vol. 49, no. 3, pp. 258-268, Sep. 2003.

[13] J. Tellado, "Peak to average power reduction for multicarrier modulation," PhD thesis (Stanford University, 2000).

[14] S. H. M"uller and J. B. Huber, "OFDM with reduced peak-to-average power ratio by optimum combination of partial transmit sequences,” IEE Elec. Letters, vol. 33, no. 5, pp. 368-369, Feb. 1997.

[15] R. W. B"auml, R. F. Fischer, and J. B. Huber, "Reducing the peak-toaverage power ratio of multicarrier modulation by selected mapping," IEE Elec. Letters, vol. 32, no. 22, pp. 2056-2057, Oct. 1996.

[16] S. H. Han and J. H. Lee, "An overview of peak-to-average power ratio reduction techniques for multicarrier transmission," IEEE Wireless Commun., vol. 12, no. 2, pp. 56-65, Apr. 2005.

[17] T. Jiang and Y. Wu, "An overview: Peak-to-average power ratio reduction techniques for OFDM signals," IEEE Trans. Broadcasting, vol. 54, no. 2, pp. 257-268, Jun. 2008.

[18] Dinesh N. Bhange, Chandrashekhar G. Dethe, "Performance of Pilot-Aided 3D-OFDM Channel Estimation using Different Antenna Configurations", Indonesian Journal of Electrical Engineering and Computer Science Vol. 8, No. 1, October 2017, pp. $77 \sim 84$ DOI: 10.11591/ijeecs.v8.i1.pp77-84.

[19] H. Tiwari, R. Roshan and R. K. Singh, "PAPR reduction in MIMO-OFDM using combined methodology of selected mapping (SLM) and partial transmit sequence (PTS)," 2014 9th International Conference on Industrial and Information Systems (ICIIS), Gwalior, 2014, pp. 1-5.

[20] S. Slimane, "Peak-to-average power ratio reduction of OFDM signals using broadband pulse shaping," in Vehicular Technology Conference, 2002. Proceedings. VTC 2002-Fall. 2002 IEEE 56th, vol. 2, 2002, pp. 889-893 vol.2.

[21] R. Reine and Z. Zang, "Analysis and comparison of a set of ISI free waveforms for PAPR reduction in OFDM systems," in TENCON 2011 - 2011 IEEE Region 10 Conference, Nov 2011, pp. 246-250.

[22] "A quadratic programming approach in pulse shaping filter design to reducing PAPR in OFDM systems," in Communications (APCC), 2013 19th Asia-Pacific Conference on, Aug 2013, pp. 572-576.

[23] M.-J. Hao and C.-H. Lai, "Pulse shaping based PAPR reduction for OFDM signals with minimum error probability," in Intelligent Signal Processing and Communications Systems, 2008. ISPACS 2008. International Symposium on, Feb 2009, pp. 1-4.

A. Aminjavaheri, A. Farhang, A. Rezazadehreyhani, L. E. Doyle and B. Farhang-Boroujeny, "OFDM Without CP in Massive MIMO," in IEEE Transactions on Wireless Communications, vol. 16, no. 11, pp. 7619-7633, Nov. 2017.

[24] G. Mahalakshmi and V. M. Bhaskaran, "Managing mobility in wireless cellular networks: A profile based approach," 2014 International Conference on Electronics and Communication Systems (ICECS), Coimbatore, 2014, pp. 1-5.

[25] Z. A. Sim, R. Reine, Z. Zang and L. Gopal, "PAPR and BER reduction in MU-MIMO-OFDM systems via a set of waveforms," 2017 IEEE International Conference on Signal and Image Processing Applications (ICSIPA), Kuching, 2017, pp. 55-60.

[26] S. Isam and I. Darwazeh, "Simple DSP-IDFT techniques for generating spectrally efficient FDM signals," 2010 7th International Symposium on Communication Systems, Networks \& Digital Signal Processing (CSNDSP 2010), Newcastle upon Tyne, 2010, pp. 20-24.

[27] 3rd Generation Partnership Project; Technical Specification Group Radio Access Network; Evolved Universal Terrestrial Radio Access (E-UTRA); Physical Layer Procedures (Release 10), 3GPP Organizational Partners TS 36.213 version 10.10.0, Jul. 2013.

[28] L. Hentil"a, P. Ky"osti, M. K"aske, M. Narandzic, and M. Alatossava, "Matlab implementation of the WINNER phase II channel model ver 1.1,” Dec. 2007. 